\title{
Minimal Residual Disease
}

National Cancer Institute

\section{Source}

National Cancer Institute. Minimal Residual Disease. NCI Thesaurus. Code C3896.

Evidence for remaining tumor following primary treatment that is only apparent using highly sensitive techniques. 